\title{
BRAUER GROUP OF MODULI OF PRINCIPAL BUNDLES OVER A CURVE
}

\author{
INDRANIL BISWAS AND YOGISH I. HOLLA
}

\begin{abstract}
Let $G$ be a semisimple linear algebraic group defined over the field $\mathbb{C}$, and let $C$ be an irreducible smooth complex projective curve of genus at least three. We compute the Brauer group of the smooth locus of the moduli space of semistable principal $G$-bundles over $C$. We also compute the Brauer group of the moduli stack of principal $G$-bundles over $C$.
\end{abstract}

\section{INTRODUCTION}

The base field will always be $\mathbb{C}$. Let $Y$ be a smooth quasiprojective variety, or more generally, a smooth algebraic stack. Using the isomorphism $\mathbb{C}^{r} \otimes \mathbb{C}^{r^{\prime}}=\mathbb{C}^{r r^{\prime}}$, we have a homomorphism $\operatorname{PGL}(r, \mathbb{C}) \times \operatorname{PGL}\left(r^{\prime}, \mathbb{C}\right) \longrightarrow \operatorname{PGL}\left(r r^{\prime}, \mathbb{C}\right)$. So a principal $\operatorname{PGL}(r, \mathbb{C})$-bundle $\mathbb{P}$ and a principal $\mathrm{PGL}\left(r^{\prime}, \mathbb{C}\right)$-bundle $\mathbb{P}^{\prime}$ on $Y$ together produce a principal $\mathrm{PGL}\left(r r^{\prime}, \mathbb{C}\right)$ bundle on $Y$, which we will denote by $\mathbb{P} \otimes \mathbb{P}^{\prime}$. The two principal bundles $\mathbb{P}$ and $\mathbb{P}^{\prime}$ are called equivalent if there are vector bundles $V$ and $V^{\prime}$ on $Y$ such that the principal bundle $\mathbb{P} \otimes \mathbb{P}(V)$ is isomorphic to $\mathbb{P}^{\prime} \otimes \mathbb{P}\left(V^{\prime}\right)$. The equivalence classes form a group which is called the Brauer group of $Y$. The addition operation is defined by the tensor product, and the inverse is defined to be the dual projective bundle. The Brauer group of $Y$ will be denoted by $\operatorname{Br}(Y)$.

Let $C$ be an irreducible smooth complex projective curve such that genus $(C) \geq 3$. Fix a line bundle $L$ over $C$ of degree $d$. Let $M(r, L)$ be the moduli space of stable vector bundles $E$ over $C$ of rank $r$ with $\bigwedge^{r} E=L$. The Brauer group $\operatorname{Br}(M(r, L))$ is cyclic of order g.c.d. $(r$, degree $(L))$ [BBGN]. A generator for $\operatorname{Br}(M(r, L))$ is obtained by restricting the universal projective bundle over $C \times M(r, L)$ to $\left\{x_{0}\right\} \times M(r, L)$, where $x_{0} \in C$ is some fixed point. The Brauer group of the smooth locus of stable principal $\operatorname{PGL}(r, \mathbb{C})$-bundles over $C$ was computed in $\mathrm{BHg}$.

Let $G$ be a semisimple linear algebraic group. Let $\mathcal{M}_{C}(G)$ be the moduli stack of principal $G$-bundles on $C$. The coarse moduli space of semistable principal $G$-bundles on $C$ will be denoted by $M_{C}^{\text {ss }}(G)$. Let

$$
M_{C}(G)^{\mathrm{rs}} \subset M_{C}^{\mathrm{ss}}(G)
$$

be the smooth locus. It is known that $M_{C}(G)^{\mathrm{rs}}$ coincides with the locus of regularly stable principal $G$-bundles on $C$ (a stable principal $G$-bundle $E_{G}$ is called regularly stable if

2000 Mathematics Subject Classification. 14F22, 14D23, 14D20.

Key words and phrases. Brauer group, principal bundle, moduli space, curve. 
the natural homomorphism from the center of $G$ to the group of automorphisms of $E_{G}$ is surjective). Our aim here is to compute the Brauer groups of $M_{C}(G)^{\text {rs }}$ and $\mathcal{M}_{C}(G)$.

We prove the following theorem (see Theorem 4.1 and Corollary 6.5):

Theorem 1.1. Assume that $G$ is semisimple and simply connected. Then the Brauer group of $\mathcal{M}_{C}(G)$ is trivial, and the Brauer group $\operatorname{Br}\left(M_{C}(G)^{\mathrm{rs}}\right)$ is the group of characters $Z_{G}^{\vee}$ of the center $Z_{G} \subset G$.

If $G$ is not simply connected, then the Brauer group of the moduli stack $\mathcal{M}_{C}(G)$ is computed in Theorem 7.4 (in conjunction with Proposition 7.3). The Brauer group of $M_{C}(G)^{\mathrm{rs}}$ is computed in Theorem 6.3 .

We will describe a corollary of these computations.

Let $Z_{\widetilde{G}}$ be the center of the universal cover $\widetilde{G}$ of $G$. Define

$$
\Psi(G) \subset \operatorname{Hom}\left(Z_{\widetilde{G}} \otimes_{\mathbb{Z}} Z_{\widetilde{G}}, \mathbb{Q} / \mathbb{Z}\right)
$$

as in (6.9). Given an element $\delta \in \pi_{1}(G) \subset Z_{\widetilde{G}}$, define

$$
\operatorname{ev}_{G}^{\delta}: \Psi(G) \longrightarrow \operatorname{Hom}\left(Z_{\widetilde{G}} / \pi_{1}(G), \mathbb{Q} / \mathbb{Z}\right)
$$

as in (6.10). The connected components of both $\mathcal{M}_{C}(G)$ and $M_{C}^{\text {ss }}(G)$ are parametrized by $\pi_{1}(G)$. For any $\delta \in \pi_{1}(G)$, let $\mathcal{M}_{C}(G)^{\delta} \subset \mathcal{M}_{C}(G)$ be the connected component corresponding to $\delta$. Let $M_{C}(G)^{\delta \text {,rs }}$ be the smooth locus of the connected component, corresponding to $\delta$, of the moduli space $M_{C}^{\text {ss }}(G)$.

We prove the following (see Corollary [7.6):

Theorem 1.2. There is a short exact sequence

$$
0 \longrightarrow \operatorname{Coker}\left(\mathrm{ev}_{G}^{\delta}\right) \longrightarrow \operatorname{Br}\left(M_{C}(G)^{\delta, \text { rs }}\right) \longrightarrow \operatorname{Br}\left(\mathcal{M}_{C}(G)^{\delta}\right) \longrightarrow 0 \text {. }
$$

The Brauer groups for the moduli spaces of principal bundles with a classical group as the structure group are computed in Section 8. As a consequence, the twisted moduli space of $\operatorname{Sp}_{2 n}(\mathbb{C})$-bundles is locally factorial if $n$ is odd, and it is not locally factorial $n$ is even. (See Corollary 8.2 .)

We note that among the exceptional groups, $G_{2}, F_{4}$ and $E_{8}$ have the property that the center is trivial. Therefore, from Theorem 1.1 it follows that for these three groups

$$
\operatorname{Br}\left(\mathcal{M}_{C}(G)\right)=0=\operatorname{Br}\left(M_{C}(G)^{\mathrm{rs}}\right) .
$$

Acknowledgements. We are grateful to the referee for providing helpful comments.

\section{Preliminaries}

As mentioned before, the base field is $\mathbb{C}$. For an algebraic stack $Y$, the torsion group $H_{\text {ét }}^{2}\left(Y, \mathbb{G}_{m}\right)_{\text {torsion }}$ is called the cohomological Brauer group of $Y$. A theorem of Grothendieck says that if $Y$ is a smooth variety, then $H_{\text {ét }}^{2}\left(Y, \mathbb{G}_{m}\right)$ is torsion [Mi]. If $Y$ is a quasiprojective variety, then a theorem of Gabber says that $H_{\text {ét }}^{2}\left(Y, \mathbb{G}_{m}\right)_{\text {torsion }}$ coincides 
with the Brauer group defined by the Morita equivalence classes of Azumaya algebras over $Y$; see [dJ, Theorem 1.1] for this theorem of Gabber.

As mentioned in the introduction, we are interested in computing the Brauer groups of the moduli stack of principal $G$-bundles as well as the smooth locus of the moduli space of semistable principal $G$-bundles over a smooth projective curve. Since the smooth locus of the moduli space is a smooth quasiprojective variety, the above theorems of Grothendieck and Gabber imply that all the above three groups associated to it coincide.

For algebraic stacks, we will only consider the cohomological Brauer group. It should be mentioned that the moduli stack of principal $G$-bundles over a smooth projective curve is smooth.

Proposition 2.1. Let $Y$ be an algebraic stack satisfying the following two properties:

- any class in $H^{2}(Y, \mathbb{Z})$ is represented by a holomorphic line bundle on $Y$, and

- each holomorphic line bundle on $Y$ admits an algebraic structure.

Then there are isomorphisms

$$
H_{e ́ t}^{2}\left(Y, \mathbb{G}_{m}\right)_{\text {torsion }} \cong H^{2}\left(Y, \mathcal{O}_{X, \text { an }}^{*}\right)_{\text {torsion }} \cong H_{B}^{3}(Y, \mathbb{Z})_{\text {torsion }}
$$

Proof. For finite coefficients, we have a comparison isomorphism between the étale cohomology and the Betti cohomology. Let $\mu_{n} \subset \mathbb{C}^{*}$ be the group of $n$-th roots of 1 . By the Kummer sequence, any element in $H_{\text {ét }}^{2}\left(Y, \mathbb{G}_{m}\right)_{\text {torsion }}$ (respectively, $\left.H_{\text {an }}^{2}\left(Y, \mathcal{O}_{Y \text {,an }}^{*}\right)_{\text {torsion }}\right)$ is represented by a class in $H_{\text {ét }}^{2}\left(Y, \mu_{n}\right)$ (respectively, $H^{2}(Y, \mathbb{Z} / n \mathbb{Z})$ ) for some $n$. This implies the surjectivity of the homomorphism

$$
H_{\text {ét }}^{2}\left(Y, \mathbb{G}_{m}\right)_{\text {torsion }} \longrightarrow H^{2}\left(Y, \mathcal{O}_{Y, \text { an }}^{*}\right)_{\text {torsion }},
$$

while its injectivity follows from the assumptions by a straight-forward diagram chase.

The second isomorphism in the proposition is derived using the exact sequence

$$
0 \longrightarrow \mathbb{Z} \longrightarrow \mathcal{O}_{Y, \text { an }} \longrightarrow \mathcal{O}_{Y, \text { an }}^{*} \longrightarrow 0
$$

associated to the homomorphism $f \longmapsto \exp (2 \pi \sqrt{-1} f)$.

See [Ne, p. 25, Theorem 1] for a similar result.

\section{The ind-Grassmannian And $L_{C}(G)$}

Let $G$ be a connected semisimple linear algebraic group over $\mathbb{C}$. Let

$$
\widetilde{G} \longrightarrow G
$$

be the universal cover. The fundamental group of $G$ will be denoted by $\pi_{1}(G)$; being abelian $\pi_{1}(G)$ is independent of the choice of base point in $G$. Let

$$
L(G):=G((t)) \quad \text { and } \quad L^{+}(G):=G[[t]]
$$

be the loop group and its naturally defined subgroup scheme respectively. Let

$$
\mathcal{Q}_{G}:=L(G) / L^{+}(G)
$$


be the ind-Grassmannian; it is a direct limit of projective integral varieties.

Let $C$ be an irreducible smooth complex projective curve with genus $(C) \geq 3$. Fix a base point $p_{0} \in C$. Let

$$
L_{C}(G):=G\left(\mathcal{O}\left(C-p_{0}\right)\right) \subset L(G)
$$

be the sub-ind-group scheme.

Let $\mathcal{M}_{C}(G)$ be the moduli stack of principal $G$-bundles on $C$. The "Uniformization theorem" produces a canonical isomorphism

$$
L_{C}(G) \backslash L(G) / L^{+}(G)=L_{C}(G) \backslash \mathcal{Q}_{G} \stackrel{\sim}{\longrightarrow} \mathcal{M}_{C}(G)
$$

[Fa], [Te], BLS].

Proposition 3.1. The cohomological Brauer group $H_{e t}^{2}\left(\mathcal{Q}_{G}, \mathcal{O}_{\mathcal{Q}_{G}}^{*}\right)_{\text {torsion }}$ of $\mathcal{Q}_{G}$ is trivial.

Proof. We begin by recalling a lemma from [BLS] (see [BLS, p. 185, Lemma 1.2]).

Lemma 3.2 ([BLS]). The quotient map $L(G) \longrightarrow \mathcal{Q}_{G}$ induces a bijection

$$
\pi_{1}(G)=\pi_{0}(L(G)) \longrightarrow \pi_{0}\left(\mathcal{Q}_{G}\right) .
$$

Each connected component of $\mathcal{Q}_{G}$ is isomorphic to $\mathcal{Q}_{\widetilde{G}}$, where $\widetilde{G}$ is the universal cover of $G$.

In view of Lemma 3.2 , it is enough to prove the proposition for simply connected groups.

First assume that the group $G$ is almost simple and simply connected.

Fix a Borel subgroup $B \subset G$ together with a maximal torus $T \subset B$ of $G$. The homomorphism of rings $\mathbb{C}[[t]] \longrightarrow \mathbb{C}$ defined by $t \longmapsto 0$ induces a homomorphism $L^{+}(G) \longrightarrow G$. Let

$$
\mathcal{B} \subset L^{+}(G)
$$

be the inverse image of the Borel subgroup $B$ under this homomorphism. We recall that $\mathcal{B}$ is called the standard Borel subgroup of $L(G)$ associated to $B$. Let $N(T)$ be the normalizer of $T$ in $G$. Let

$$
\widetilde{W}:=\operatorname{Mor}\left(\mathbb{C}^{*}, N(T)\right) / T
$$

be the affine Weyl group containing the Weyl group $W=N(T) / T$ as a subgroup (the constant morphisms from $\mathbb{C}^{*}$ to $N(T)$ make $N(T)$ a subgroup of $\left.\operatorname{Mor}\left(\mathbb{C}^{*}, N(T)\right)\right)$.

The ind-Grassmannian $\mathcal{Q}_{G}$ has the Bruhat decomposition

$$
\mathcal{Q}_{G}=\bigcup_{w \in \widetilde{W} / W} \mathcal{B} w^{\prime} L^{+}(G) / L^{+}(G),
$$

where $w^{\prime} \in \widetilde{W}$ is any chosen representative of $w$. The quotient space $\widetilde{W} / W$ has a partial ordering inherited from the Bruhat partial ordering of the affine Weyl group $\widetilde{W}$. This makes $\mathcal{Q}_{G}$ a direct limit of projective varieties $\left\{Q_{w}\right\}_{w \in \widetilde{W} / W}$, where

$$
Q_{w}:=\bigcup_{v \leq w} \mathcal{B} v L^{+}(G) / L^{+}(G)
$$


For any $w \in \widetilde{W} / W$, consider the length of every element in the coset $w$. The smallest one among them will be denoted by $\ell(w)$.

The generalized Schubert variety $Q_{w}$ in (3.2) has a Zariski open subset defined by $\mathcal{B} w L^{+}(G) / L^{+}(G)$ which is biregularly isomorphic with the affine space $\mathbb{A}^{\ell(w)}$, where $\ell(w)$ is defined above. As a result, $Q_{w}$ equipped with the analytic topology has the structure of a CW complex with only even dimensional cells. Hence $H^{3}\left(Q_{w}, \mathbb{Z}\right)=0$. This implies that

$$
H^{3}\left(\mathcal{Q}_{G}, \mathbb{Z}\right)=0
$$

because $\mathcal{Q}_{G}$ is a direct limit of these varieties $Q_{w}$. In particular,

$$
H^{3}\left(Q_{w}, \mathbb{Z}\right)_{\text {torsion }}=0=H^{3}\left(\mathcal{Q}_{G}, \mathbb{Z}\right)_{\text {torsion }}
$$

Similarly, we have

$$
H^{1}\left(\mathcal{Q}_{G}, \mathbb{Z}\right)=0
$$

Both $Q_{w}$ and $\mathcal{Q}_{G}$ satisfy the two assumptions in Proposition 2.1 [KN, p. 157, Lemma 2.2]. Therefore, the proposition follows from Proposition 2.1 and (3.3) under the assumption that $G$ is almost simple and simply connected.

In the general case where $G$ is semisimple and simply connected, write $G$ as a product

$$
G=\prod_{i=1}^{s} G_{i},
$$

where each $G_{i}$ is almost simple and simply connected. This enables us to write the ind-Grassmannian $\mathcal{Q}_{G}$ as a product

$$
\mathcal{Q}_{G}=\prod_{i=1}^{s} \mathcal{Q}_{G_{i}},
$$

where $\mathcal{Q}_{G_{i}}$ is the ind-Grassmannian for $G_{i}$. In view of (3.3) and (3.4) for $G_{i}$, from the Künneth decomposition of $H^{3}\left(\prod_{i=1}^{s} \mathcal{Q}_{G_{i}}, \mathbb{Z}\right)$ we conclude that $H^{3}\left(\mathcal{Q}_{G}, \mathbb{Z}\right)_{\text {torsion }}=0$. The two conditions in Proposition 2.1 hold for $\mathcal{Q}_{G}[\mathrm{KN}$, p. 157, Lemma 2.2]. Now the proof of the proposition is completed using Proposition 2.1.

3.1. Some properties of $L_{C}(G)$. Let $G$ be semisimple and simply connected. The topological properties of the ind-group scheme $L_{C}(G)$ that we need can be derived from the following theorem of Teleman ([Te, p. 8, Theorem 1]):

Theorem 3.3 ([Te]). The natural map $L_{C}(G) \longrightarrow C^{\infty}\left(C \backslash\left\{p_{0}\right\}, G\right)$ defines a homotopy equivalence. Hence the homotopy type of $L_{C}(G)$, equipped with the analytic topology, is that of $G \times \Omega G^{2 g}$.

The connectedness and simply connectedness of $L_{C}(G)$ follow immediately from Theorem 3.3 .

Proposition 3.4. Let $G$ be semisimple and simply connected. Let $B L_{C}(G)$ be the classifying space for $L_{C}(G)$. Then 
(1) $H^{1}\left(B L_{C}(G), \mathbb{Z}\right)=0$,

(2) $H^{2}\left(B L_{C}(G), \mathbb{Z} / n \mathbb{Z}\right)=0$ for all $n$, and

(3) $H^{2}\left(B L_{C}(G), \mathbb{C}^{*}\right)=0$.

Proof. The group $H^{1}\left(B L_{C}(G), \mathbb{Z}\right)$ parametrizes the space of all continuous homomorphisms from $L_{C}(G)$ to $\mathbb{Z}$. So $H^{1}\left(B L_{C}(G), \mathbb{Z}\right)$ is trivial by the connectedness of $L_{C}(G)$.

The connectedness of $L_{C}(G)$ also implies that $\pi_{1}\left(B L_{C}(G)\right)=\pi_{0}\left(L_{C}(G)\right)=0$. The simply connectedness of $L_{C}(G)$ implies that $\pi_{2}\left(B L_{C}(G)\right)=\pi_{1}\left(L_{C}(G)\right)=0$. Hence statements (2) and (3) of the proposition follow from the Hurewicz's theorem.

\section{Brauer group of MOduli: $G$ IS Simply CONNECTED}

Theorem 4.1. Let $G$ be simply connected and semisimple. Then $\operatorname{Br}\left(\mathcal{M}_{C}(G)\right)=0$.

Proof. The descent spectral sequence for the principal $L_{C}(G)$-bundle $\mathcal{Q}_{G} \longrightarrow \mathcal{M}_{C}(G)$ gives the following exact sequence in the analytic topology:

$$
\begin{gathered}
0 \longrightarrow H^{1}\left(B L_{C}(G), \mathbb{C}^{*}\right) \longrightarrow H^{1}\left(\mathcal{M}_{C}(G), \mathcal{O}^{*}\right) \stackrel{\theta}{\longrightarrow} H^{0}\left(B L_{C}(G), H^{1}\left(\mathcal{Q}_{G}, \mathcal{O}^{*}\right)\right) \\
\longrightarrow H^{2}\left(B L_{C}(G), \mathbb{C}^{*}\right) \longrightarrow \operatorname{kernel}\left[H^{2}\left(\mathcal{M}_{C}(G), \mathcal{O}^{*}\right) \longrightarrow H^{0}\left(B L_{C}(G), H^{2}\left(\mathcal{Q}_{G}, \mathcal{O}^{*}\right)\right)\right] \\
\longrightarrow H^{1}\left(B L_{C}(G), H^{1}\left(\mathcal{Q}_{G}, \mathcal{O}^{*}\right)\right) ;
\end{gathered}
$$

cf. [Me, p. 371, Corollary 3.2] (see also [Te, p. 10, (1.9)], [Te, p. 27, (5.5)]).

Let $s$ be the number of almost simple factors in the product decomposition of $G$. We have $H_{\text {êt }}^{2}\left(\mathcal{Q}_{G}, \mathcal{O}_{\mathcal{Q}_{G}}^{*}\right)_{\text {torsion }}=0$ by Proposition 3.1. Also, $H^{1}\left(\mathcal{Q}_{G}, \mathcal{O}^{*}\right) \cong \mathbb{Z}^{s}$ BLS, p. 186, Lemma 1.4]. Therefore, from Proposition 3.4(1),

$$
H^{0}\left(B L_{C}(G), H^{2}\left(\mathcal{Q}_{G}, \mathcal{O}_{\mathcal{Q}_{G}}^{*}\right)\right)=0=H^{1}\left(B L_{C}(G), H^{1}\left(\mathcal{Q}_{G}, \mathcal{O}_{\mathcal{Q}_{G}}^{*}\right)\right) \text {. }
$$

The homomorphism of the Picard groups

$$
\theta: \operatorname{Pic}\left(\mathcal{M}_{C}(G)\right) \longrightarrow \operatorname{Pic}\left(\mathcal{Q}_{G}\right) \cong \mathbb{Z}^{s}
$$

in (4.1) has a finite cokernel [BLS, p. 187, Proposition 1.5], in particular, this cokernel is a torsion group. Since $H^{2}\left(B L_{C}(G), \mathbb{C}^{*}\right)=0$ (see Proposition 3.4(3)), from (4.1) it follows that $\theta$ in (4.3) is actually surjective. Therefore, using (4.2), from (4.1) we conclude that

$$
H^{2}\left(\mathcal{M}_{C}(G), \mathcal{O}^{*}\right)=H^{2}\left(B L_{C}(G), \mathbb{C}^{*}\right)=0 .
$$

Now we apply Proposition 2.1 to the stack $\mathcal{M}_{C}(G)$; from [Te, p. 26, Proposition 5.1] and [Te, p. 26, Remark 5.2] it follows that the two conditions in Proposition 2.1 are satisfied. Therefore, we conclude that $\operatorname{Br}\left(\mathcal{M}_{C}(G)\right)=0$.

Remark 4.2. It should be pointed out that the above arguments involving sheaves in analytic topology can be replaced by an argument which uses only constant sheaves in Euclidean topology. Using the exponential sequence, the Brauer group $\operatorname{Br}(X)$ for any $X$ can be expressed as the quotient of $H^{2}\left(X, \mathbb{C}^{*}\right)$ by the image of $H^{2}(X, \mathbb{C})$. The isomorphism

$$
H^{2}\left(\mathcal{M}_{C}(G), \mathbb{C}\right) \cong H^{2}(\mathcal{Q}(G), \mathbb{C})
$$


simplifies the terms in the descent spectral sequence associated to the constant sheaf $\mathbb{C}^{*}$.

Let

$$
Z_{G} \subset G
$$

be the center. Let $M_{C}^{\text {ss }}(G)$ be the coarse moduli space of semistable principal $G$-bundles over $C$. The smooth locus of $M_{C}^{\text {ss }}(G)$ is the locus of regularly stable principal $G$-bundles over $C$ [BHf2, Corollary 3.6]. We recall that a principal $G$-bundle $E$ is called regularly stable if $E$ is stable, and the natural homomorphism $Z_{G} \longrightarrow \operatorname{Aut}(\mathrm{E})$ is surjective (the restriction to $Z_{G}$ of the action of $G$ on $E$ produces this homomorphism). Let

$$
\mathcal{M}_{C}(G)^{\mathrm{rs}} \subset \mathcal{M}_{C}(G)
$$

be the open sub-stack defined by the regularly stable bundles [BHf1, Lemma 2.3]. Let

$$
p: \mathcal{M}_{C}(G)^{\mathrm{rs}} \longrightarrow M_{C}(G)^{\mathrm{rs}}
$$

be the morphism to the coarse moduli space, so $M_{C}(G)^{\mathrm{rs}}$ is the moduli space of regularly stable principal $G$-bundles over $C$. As noted above, $M_{C}(G)^{\text {rs }}$ is the smooth locus of $M_{C}^{\mathrm{ss}}(G)$.

Proposition 4.3. As before, $G$ is semisimple.

(1) $\mathcal{M}_{C}(G)^{\mathrm{rs}}$ is an open sub-stack of the moduli stack $\mathcal{M}_{C}(G)$, and the complement of it in $\mathcal{M}_{C}(G)$ is of codimension at least two.

(2) The morphism $p$ in (4.4) defines a gerbe over $M_{C}(G)^{\mathrm{rs}}$ banded by $Z_{G}$.

Proof. See [Fa, Theorem II.6] or Theorem 2.4 of [BHf1] for a proof of the first part. The second part is proved in Section 6 of [BHf1].

Let

$$
\psi \in H^{2}\left(M_{C}(G)^{\mathrm{rs}}, Z_{G}\right)
$$

be the cohomology class defined by the gerbe in (4.4).

Let $Z$ be a finite abelian group, and let

$$
\alpha: \mathcal{M} \longrightarrow M
$$

be a gerbe banded by $Z$, where $M$ is irreducible. Take any line bundle $L$ over $\mathcal{M}$. So $L$ is given by a functor $L_{S}$ from $\mathcal{M}(S)$ to the groupoid of line bundles on $S$ for every $\mathbb{C}$-scheme $S$. In particular, $L_{S}$ defines for every object $\mathcal{E}$ in $\mathcal{M}(S)$ a group homomorphism

$$
L_{S, \mathcal{E}}: \operatorname{Aut}_{\mathcal{M}(S)}(\mathcal{E}) \longrightarrow \operatorname{Aut}_{\mathcal{O}_{S}}\left(L_{S}(\mathcal{E})\right)=\Gamma\left(S, \mathcal{O}_{S}^{*}\right)
$$

The compatibility conditions ensure that the composition

$$
Z(S) \stackrel{\iota_{\mathcal{E}}}{\longrightarrow} \operatorname{Aut}_{\mathcal{M}(S)}(\mathcal{E}) \stackrel{L_{S, \mathcal{E}}}{\longrightarrow} \Gamma\left(S, \mathcal{O}_{S}^{*}\right)
$$

defines a 1 -morphism $Z \times \mathcal{M} \longrightarrow \mathbb{G}_{m} \times \mathcal{M}$ over $\mathcal{M}$. As $M$ is connected, and $\operatorname{Hom}\left(Z, \mathbb{G}_{m}\right)$ is discrete, this 1 -morphism is the pullback of some character $\chi: Z \longrightarrow \mathbb{G}_{m}$. We call $\chi$ the weight of $L$. (See [BHf1, Section 6].) 
Lemma 4.4. Let $\beta \in H^{2}(M, Z)$ be the class of the gerbe in (4.6) banded by $Z$. Then there is an exact sequence

$$
0 \longrightarrow \operatorname{Pic}(M) \stackrel{\alpha^{*}}{\longrightarrow} \operatorname{Pic}(\mathcal{M}) \stackrel{\text { wt }}{\longrightarrow} \operatorname{Hom}\left(Z, \mathbb{G}_{m}\right) \stackrel{\beta_{*}}{\longrightarrow} \operatorname{Br}(M) \stackrel{\alpha^{*}}{\longrightarrow} \operatorname{Br}(\mathcal{M}),
$$

where $\beta_{*}$ takes any homomorphism $\eta: Z \longrightarrow \mathbb{G}_{m}$ to the image of $\beta$ under the homomorphism $H^{2}(M, Z) \longrightarrow H^{2}\left(M, \mathbb{G}_{m}\right)$ induced by $\eta$.

Proof. This is the Leray spectral sequence for $\mathcal{O}_{\mathcal{M}}^{*} \longrightarrow \mathcal{M} \stackrel{\alpha}{\longrightarrow} M$. The only points to note are that

$$
\alpha_{*} \mathcal{O}_{\mathcal{M}}^{*}=\mathcal{O}_{M}^{*}
$$

and $R^{1} \alpha_{*} \mathcal{O}_{\mathcal{M}}^{*}$ is the constant $\operatorname{sheaf} \operatorname{Hom}\left(Z, \mathbb{G}_{m}\right)$.

Theorem 4.5. There is an exact sequence

$$
0 \longrightarrow \mathbb{Z}^{s} \stackrel{p^{*}}{\longrightarrow} \mathbb{Z}^{s} \stackrel{\mathrm{wt}}{\longrightarrow} \operatorname{Hom}\left(Z_{G}, \mathbb{G}_{m}\right) \stackrel{\psi_{*}}{\longrightarrow} \operatorname{Br}\left(M_{C}(G)^{\mathrm{rs}}\right) \longrightarrow 0,
$$

where $s$ is the number of almost simple factors in $G, p^{*}$ is the homomorphism of Picard groups induced by the morphism $p$ in (4.4), wt is the weight map defined above, and $\psi_{*}$ is constructed using the class $\psi$ in (4.5) in the obvious way.

Proof. From Theorem 4.1 and Proposition $4.3(1)$ it follows that $\operatorname{Br}\left(\mathcal{M}_{C}(G)^{\mathrm{rs}}\right)=0$. Therefore, Lemma 4.4 applied to the gerbe in (4.4) produces the exact sequence.

\section{THE TWISTED CASE}

In this section the semisimple group $G$ is assumed to be simply connected but the moduli stack and moduli space will be twisted (defined below).

Fix a maximal torus $T$ of $G$. As before, the center of $G$ will be denoted by $Z_{G}$. Let $\sigma$ be the rank of $G$, so $\sigma$ is the dimension of $T$.

As before, for any $m$, let $\mu_{m} \subset \mathbb{C}^{*}$ be the group of all $m$-th roots of 1 . We fix an isomorphism

$$
\rho: T \longrightarrow\left(\mathbb{G}_{m}\right)^{\sigma} \quad \text { with } \rho\left(Z_{G}\right)=\prod_{i=1}^{\sigma} \mu_{r_{i}} .
$$

Using this isomorphism $\rho$, the homomorphism

$$
\left(\mathbb{G}_{m}\right)^{\sigma} \longrightarrow\left(\mathbb{G}_{m}\right)^{\sigma}
$$

defined by $\prod_{i=1}^{\sigma} z_{i} \longmapsto \prod_{i=1}^{\sigma}\left(z_{i}\right)^{r_{i}}$ produces an isomorphism

$$
T / Z_{G}=\left(\mathbb{G}_{m}\right)^{\sigma} / \rho\left(Z_{G}\right) \stackrel{\sim}{\longrightarrow} T .
$$

Let $C(G)$ denote the quotient of $G \times T$ by $Z_{G}$ for the diagonal action. The projections

$$
q: C(G) \longrightarrow T / Z_{G}=T \text { and } p: C(G) \longrightarrow G / Z_{G}
$$

induce morphisms of stacks

$$
\text { det }: \mathcal{M}_{C}(C(G)) \longrightarrow \mathcal{M}_{C}(T)
$$


and

$$
\mathcal{M}_{C}(C(G)) \longrightarrow \mathcal{M}_{C}\left(G / Z_{G}\right)
$$

respectively.

For an element $\mathbf{d}=\left(d_{1}, \ldots, d_{\sigma}\right) \in \mathbb{Z}^{\sigma}$, let $\mathcal{O}_{C}(\mathbf{d} p)$ denote the rational point of $\mathcal{M}_{C}(T)$ defined by $\left(\mathcal{O}_{C}\left(d_{1} p_{0}\right), \ldots, \mathcal{O}_{C}\left(d_{\sigma} p_{0}\right)\right)$. Define

$$
\mathcal{M}_{C}^{\mathbf{d}}(G):=\operatorname{det}^{-1}\left(\mathcal{O}_{C}(\mathbf{d} p)\right) \subset \mathcal{M}_{C}(C(G)),
$$

where det is the morphism in (5.2). Let

$$
\Phi: \mathcal{M}_{C}^{\mathbf{d}}(G) \longrightarrow \mathcal{M}_{C}\left(G / Z_{G}\right)
$$

be the restriction of the morphism in (5.3).

Take any $\delta \in Z_{G}$. Consider

$$
\rho(\delta)=\left(a_{1}, \cdots, a_{\sigma}\right) \in\left(\mathbb{G}_{m}\right)^{\sigma},
$$

where $\rho$ is the homomorphism in (5.1). Take $\mathbf{d} \in \mathbb{Z}^{\sigma}$ such that there is an element

$$
\mathbf{r}:=\left(r_{1}, \ldots, r_{\sigma}\right) \in \mathbb{Z}^{\sigma}
$$

satisfying the condition that $\exp \left(-2 \pi \sqrt{-1} d_{i} / r_{i}\right)=a_{i}$ for every $i \in[1, \sigma]$.

The connected components of $\mathcal{M}_{C}\left(G / Z_{G}\right)$ are parametrized by $\pi_{1}\left(G / Z_{G}\right)=Z_{G}$ (see Lemma 3.2); recall that $G$ is simply connected. Let

$$
\mathcal{M}_{C}\left(G / Z_{G}\right)^{\delta} \subset \mathcal{M}_{C}\left(G / Z_{G}\right)
$$

be the connected component corresponding to the element $\delta$. Let

$$
\mathcal{M}_{C}^{\delta}(G):=\Phi^{-1}\left(\mathcal{M}_{C}\left(G / Z_{G}\right)^{\delta}\right) \subset \mathcal{M}_{C}^{\mathrm{d}}(G)
$$

be the open and closed sub-stack of $\mathcal{M}_{C}^{\mathrm{d}}(G)$, where $\Phi$ is the morphism in (5.5). This $\mathcal{M}_{C}^{\delta}(G)$ is called the twisted moduli stack (see [BLS, Section 2]).

The restriction

$$
\left.\Phi\right|_{\mathcal{M}_{C}^{\delta}(G)}: \mathcal{M}_{C}^{\delta}(G) \longrightarrow \mathcal{M}_{C}\left(G / Z_{G}\right)^{\delta}
$$

is surjective.

The group $G / Z_{G}$ will also be denoted by $G_{\mathrm{ad}}$. The connected components of $L\left(G_{\mathrm{ad}}\right)$ are parametrized by $Z_{G}$ (see Lemma 3.2). Let

$$
\left(L G_{\mathrm{ad}}\right)^{\delta} \subset L\left(G_{\mathrm{ad}}\right)
$$

be the connected component corresponding to $\delta$.

The following proposition is proved in [BLS] (see [BLS, p. 189, (2.4)]):

Proposition $5.1([\mathrm{BLS}])$. For any $\delta \in Z_{G}$, and any $\zeta \in\left(L G_{\mathrm{ad}}\right)^{\delta}(\mathbb{C})$, where $\left(L G_{\mathrm{ad}}\right)^{\delta}$ is constructed in (5.7), there is a natural isomorphism

$$
\mathcal{M}_{C}^{\delta}(G) \cong\left(\zeta^{-1} L_{C}(G) \zeta\right) \backslash \mathcal{Q}_{\widetilde{G}}
$$


Proposition 5.1 implies that the of proof of Theorem 4.1 goes through in the twisted case being considered. So we have the following theorem:

Theorem 5.2. For any $\delta \in Z_{G}$, the group $\operatorname{Br}\left(\mathcal{M}_{C}^{\delta}(G)\right)$ is trivial.

There is a coarse moduli space $M_{C}^{\delta}(G)^{\text {ss }}$ for the open sub-stack of $\mathcal{M}_{C}^{\delta}(G)$ defined by the locus of semistable principal $C(G)$-bundles. There is also an open subscheme $M_{C}^{\delta}(G)^{\mathrm{rs}} \subset M_{C}^{\delta}(G)^{\text {ss }}$ corresponding to the regularly stable principal $C(G)$-bundles.

Proposition 5.3. The codimension of the complement of $\mathcal{M}_{C}^{\delta}(G)^{\mathrm{rs}}$ in $\mathcal{M}_{C}^{\delta}(G)$ is at least two.

The smooth locus of $M_{C}^{\delta}(G)^{\mathrm{ss}}$ is $M_{C}^{\delta}(G)^{\mathrm{rs}}$.

Proof. Let $\mathcal{M}_{C}^{\delta}\left(G / Z_{G}\right)^{\text {rs }}$ be the sub-stack of $\mathcal{M}_{C}^{\delta}\left(G / Z_{G}\right)$ corresponding to the regularly stable principal $C(G)$-bundles; that it is a sub-stack follows from [BHf1, Lemma 2.3]. By [Fa, Theorem II.6] or [BHf1, Theorem 2.4] it follows that the complement of $\mathcal{M}_{C}^{\delta}\left(G / Z_{G}\right)^{\text {rs }}$ in $\mathcal{M}_{C}^{\delta}\left(G / Z_{G}\right)$ has codimension at least two. Taking inverse image for the morphism $\left.\Phi\right|_{\mathcal{M}_{C}^{\delta}(G)}$ in (5.6), the first part of the proposition follows.

The proof that the smooth locus of $M_{C}^{\delta}(G)^{\mathrm{ss}}$ is $M_{C}^{\delta}(G)^{\mathrm{rs}}$ is identical to that of [BHf2, Corollary 3.6].

The morphism to the coarse moduli space

$$
p: \mathcal{M}_{C}^{\delta}(G)^{\mathrm{rs}} \longrightarrow M_{C}^{\delta}(G)^{\mathrm{rs}}
$$

defines a gerbe banded by $Z_{G}$. Let

$$
\psi \in H^{2}\left(M_{C}^{\delta}(G)^{\mathrm{rs}}, Z_{G}\right)
$$

be the class of this gerbe.

Just like Theorem 4.5, we now have the following moduli space version of Theorem 5.2 ,

Theorem 5.4. There is an exact sequence

$$
0 \longrightarrow \operatorname{Pic}\left(M_{C}^{\delta}(G)^{\mathrm{rs}}\right) \stackrel{p^{*}}{\longrightarrow} \operatorname{Pic}\left(\mathcal{M}_{C}^{\delta}(G)^{\mathrm{rs}}\right) \stackrel{\mathrm{wt}}{\longrightarrow} \operatorname{Hom}\left(Z_{G}, \mathbb{G}_{m}\right) \stackrel{\psi_{*}}{\longrightarrow} \operatorname{Br}\left(M_{C}^{\delta}(G)^{\mathrm{rs}}\right) \longrightarrow 0,
$$

where $p$ is the morphism in (5.8), wt is the weight defined earlier, and $\psi_{*}$ takes a homomorphism $\eta: Z_{G} \longrightarrow \mathbb{G}_{m}$ to the image of $\psi$ under the homomorphism $H^{2}(M, Z) \longrightarrow$ $H^{2}\left(M, \mathbb{G}_{m}\right)$ induced by $\eta$.

\section{Brauer Group of MOduli: $G$ IS NOT SIMPly CONNECTED}

In this section we compute the Brauer group of the moduli stack and also that of the smooth locus of the moduli space of principal $G$-bundles when the semisimple group $G$ is not necessarily simply connected.

Let $\widetilde{G}$ be the universal cover of the semisimple group $G$. The fundamental group $\pi_{1}(G)$ is a subgroup of the center

$$
Z_{\widetilde{G}} \subset \widetilde{G}
$$


Fix a maximal torus $\widetilde{T} \subset \widetilde{G}$. We fix an isomorphism

$$
\rho: \widetilde{T} \longrightarrow\left(\mathbb{G}_{m}\right)^{\sigma} \quad \text { with } \rho\left(\pi_{1}(G)\right)=\prod_{i=1}^{\sigma} \mu_{r_{i}} .
$$

There is a canonical isomorphism $\pi_{0}\left(\mathcal{M}_{C}(G)\right)=\pi_{0}(L(G)) \cong \pi_{1}(G)$. Take any

$$
\delta \in \pi_{1}(G) \text {. }
$$

Let

$$
L(G)^{\delta} \subset L(G) \text { and } \mathcal{M}_{C}(G)^{\delta} \subset \mathcal{M}_{C}(G)
$$

be the connected components corresponding to $\delta$.

The above notation $\mathcal{M}_{C}(G)^{\delta}$ for a connected component should not be confused with the previous notation $\mathcal{M}_{C}^{\delta}(G)$ for a twisted moduli stack. It should also be clarified that the twisted moduli stack (or the twisted moduli space) is defined only for simply connected groups.

The following proposition is proved in [BLS]; see [BLS, p. 186, Proposition 1.3].

Proposition 6.1. Take any $\delta \in \pi_{1}(G)$. For any $\zeta \in L(G)^{\delta}(\mathbb{C})$, there is a natural isomorphism

$$
\mathcal{M}_{C}(G)^{\delta} \cong\left(\zeta^{-1} L_{C}(G) \zeta\right) \backslash \mathcal{Q}_{\widetilde{G}} .
$$

Let

$$
q_{G}^{\delta}: \mathcal{Q}_{\widetilde{G}} \longrightarrow \mathcal{M}_{C}(G)^{\delta}
$$

be the quotient morphism in Proposition 6.1.

Let

$$
\widehat{q}_{\widetilde{G}}^{\delta}: \mathcal{Q}_{\widetilde{G}} \longrightarrow \mathcal{M}_{C}^{\delta}(\widetilde{G})
$$

be the quotient morphism to the twisted moduli stack in Proposition 5.1. There is a natural morphism of stacks

$$
\gamma: \mathcal{M}_{C}^{\delta}(\widetilde{G}) \longrightarrow \mathcal{M}_{C}(G)^{\delta} .
$$

This morphism $\gamma$ takes the semistable locus in $\mathcal{M}_{C}^{\delta}(\widetilde{G})$ to the semistable locus in $\mathcal{M}_{C}(G)^{\delta}$; this is an immediately consequence of [Ra, p. 319, Proposition 3.17]. Hence $\gamma$ induces a morphism

$$
\gamma_{1}: M_{C}^{\delta}(\widetilde{G}) \longrightarrow M_{C}(G)^{\delta}
$$

between the corresponding coarse moduli spaces of semistable bundles.

Let

$$
M_{C}(G)^{\delta, \mathrm{rs}} \subset M_{C}(G)^{\delta}
$$

be the smooth locus; we recall that $M_{C}(G)^{\delta \text {,rs }}$ parametrizes the regularly stable bundles in $M_{C}(G)^{\delta}$ (see [BHf2, Corollary 3.6]). Define the finite group

$$
\Gamma:=H^{1}\left(C, \pi_{1}(G)\right) .
$$


Lemma 6.2. (1) The morphism $q_{G}^{\delta}$ in (6.1) factors as follows:

$$
\mathcal{Q}_{\widetilde{G}} \stackrel{\widehat{q}_{\widetilde{G}}^{\delta}}{\longrightarrow} \mathcal{M}_{C}^{\delta}(\widetilde{G}) \stackrel{\gamma}{\longrightarrow} \mathcal{M}_{C}(G)^{\delta},
$$

where $\widehat{q}_{\widetilde{G}}^{\delta}$ and $\gamma$ are defined in (6.2) and (6.3) respectively.

(2) The restriction of the morphism $\gamma_{1}$ in (6.4) to the Zariski open subset

$$
\gamma_{1}^{-1}\left(M_{C}(G)^{\delta, \mathrm{rs}}\right) \subset M_{C}^{\delta}(\widetilde{G})^{\mathrm{rs}}
$$

defines a principal $\Gamma$-bundle over $M_{C}(G)^{\delta, \mathrm{rs}}$, where $\Gamma$ is defined in (6.5).

Proof. See [BLS, p. 189, (2.4)] for the first part. The second part follows from the definition of regularly stable bundles.

Theorem 6.3. The Brauer group computation for $M_{C}(G)^{\delta, \mathrm{rs}}$ and $M_{C}^{\delta}(\widetilde{G})^{\mathrm{rs}}$ are related by the following exact sequence:

$$
\begin{aligned}
0 \longrightarrow H^{1}\left(\Gamma, \mathbb{C}^{*}\right) \longrightarrow H^{1}\left(M_{C}(G)^{\delta, \mathrm{rs}}, \mathcal{O}^{*}\right) \longrightarrow H^{1}\left(M_{C}^{\delta}(\widetilde{G})^{\mathrm{rs}}, \mathcal{O}^{*}\right) \\
\longrightarrow H^{2}\left(\Gamma, \mathbb{C}^{*}\right) \longrightarrow \operatorname{Br}\left(M_{C}(G)^{\delta, \mathrm{rs}}\right) \longrightarrow \operatorname{Br}\left(M_{C}^{\delta}(\widetilde{G})^{\mathrm{rs}}\right) \longrightarrow 0
\end{aligned}
$$

Proof. Consider the open subset $\gamma_{1}^{-1}\left(M_{C}(G)^{\delta, \mathrm{rs}}\right) \subset M_{C}^{\delta}(\widetilde{G})^{\mathrm{rs}}$ in Lemma 6.2. We will show that its complement is of codimension at least two. For that first note that the morphism $\gamma_{1}$ in (6.4) is finite because for any ample line bundle $L_{0}$ on $M_{C}^{\delta}(\widetilde{G})^{\text {rs }}$, the pullback $\gamma_{1}^{*} L_{0}$ is ample. Therefore, the fact that the codimension of the complement of the open subset $M_{C}(G)^{\delta, \text { rs }} \subset M_{C}(G)$ is at least two implies that the codimension of the complement of $\gamma_{1}^{-1}\left(M_{C}(G)^{\delta, \mathrm{rs}}\right)$ is at least two.

We can apply the Serre spectral sequence to the principal $\Gamma$-bundle

$$
\gamma_{1}: \gamma_{1}^{-1}\left(M_{C}(G)^{\delta, \mathrm{rs}}\right) \subset M_{C}^{\delta}(\widetilde{G})^{\mathrm{rs}}
$$

in Lemma 6.2 and get the following exact sequence:

$$
\begin{aligned}
0 \longrightarrow & H^{1}\left(\Gamma, \mathbb{C}^{*}\right) \longrightarrow H^{1}\left(M_{C}(G)^{\delta, \mathrm{rs}}, \mathcal{O}^{*}\right) \longrightarrow H^{1}\left(M_{C}^{\delta}(\widetilde{G})^{\mathrm{rs}}, \mathcal{O}^{*}\right) \\
& \longrightarrow H^{2}\left(\Gamma, \mathbb{C}^{*}\right) \longrightarrow \operatorname{Br}\left(M_{C}(G)^{\delta, \mathrm{rs}}\right) \longrightarrow \operatorname{Br}\left(M_{C}^{\delta}(\widetilde{G})^{\mathrm{rs}}\right) .
\end{aligned}
$$

It remains to show that the above homomorphism

$$
\operatorname{Br}\left(M_{C}(G)^{\delta, \mathrm{rs}}\right) \longrightarrow \operatorname{Br}\left(M_{C}^{\delta}(\widetilde{G})^{\mathrm{rs}}\right)
$$

is surjective.

Let $\mathcal{M}_{C}^{\delta}(\widetilde{G})^{\prime} \subset \mathcal{M}_{C}^{\delta}(\widetilde{G})^{\text {rs }}$ be the open sub-stack that lies over $\gamma_{1}^{-1}\left(M_{C}(G)^{\delta, \text { rs }}\right)$. The moduli stack $\mathcal{M}_{C}\left(\pi_{1}(G)\right)$ of principal $\pi_{1}(G)$-bundles on $C$ acts naturally on $\mathcal{M}_{C}^{\delta}(\widetilde{G})^{\prime}$. We note that $\mathcal{M}_{C}\left(\pi_{1}(G)\right)$ is a gerbe over $\Gamma$ (defined in (6.5) ) banded by $\pi_{1}(G)$. Since $\Gamma$ is a finite group, this gerbe is isomorphic to $\Gamma \times B\left(\pi_{1}(G)\right)$. Fixing such an isomorphism, we construct an action of $\Gamma$ on $\mathcal{M}_{C}^{\delta}(\widetilde{G})^{\prime}$ by restricting the action of $\mathcal{M}_{C}\left(\pi_{1}(G)\right)$. The quotient for this action

$$
\mathcal{M}_{C}^{\delta}(\widetilde{G})^{\prime} / \Gamma \longrightarrow \gamma_{1}^{-1}\left(M_{C}(G)^{\delta, \mathrm{rs}}\right) / \Gamma=M_{C}(G)^{\delta, \mathrm{rs}}
$$

is a gerbe banded by $\pi_{1}(G)$. 
Recall that the homomorphism $\psi_{*}$ in Theorem 5.4 is surjective, so $\operatorname{Br}\left(\mathcal{M}_{C}^{\delta}(\widetilde{G})^{\mathrm{rs}}\right)$ is constructed from the gerbe

$$
\mathcal{M}_{C}^{\delta}(\widetilde{G})^{\mathrm{rs}} \longrightarrow M_{C}^{\delta}(\widetilde{G})^{\mathrm{rs}}
$$

(which is banded by $Z_{\widetilde{G}}$ ) using $\operatorname{Hom}\left(Z_{\widetilde{G}}, \mathbb{G}_{m}\right)$. We have shown that this gerbe descends to $M_{C}(G)^{\delta \text {,rs }}$. Hence the homomorphism in (6.6) is surjective.

Let $\mathcal{M}_{C}(G)^{\delta \text {,rs }}$ be the open sub-stack of $\mathcal{M}_{C}(G)^{\delta}$ defined by the regularly stable principal bundles. The morphism to the coarse moduli space

$$
p: \mathcal{M}_{C}(G)^{\delta, \mathrm{rs}} \longrightarrow M_{C}(G)^{\delta, \mathrm{rs}}
$$

is a gerbe banded by $Z_{G}$, and we have the induced homomorphism

$$
p^{*}: \operatorname{Br}\left(M_{C}(G)^{\delta, \mathrm{rs}}\right) \longrightarrow \operatorname{Br}\left(\mathcal{M}_{C}(G)^{\delta}\right) .
$$

Now the exact sequence in Lemma 4.4 can be used in relating the Brauer group of the smooth locus of the moduli space to the Brauer group of the stack. The kernel of the homomorphism $p^{*}$ in (6.8) can be computed using a result of [BHf1] which will be recalled below.

Let $\Psi \subset \operatorname{Hom}\left(Z_{\widetilde{G}} \otimes_{\mathbb{Z}} Z_{\widetilde{G}}, \mathbb{Q} / \mathbb{Z}\right)$ be the abelian group of all symmetric bilinear maps

$$
b: Z_{\widetilde{G}} \times Z_{\widetilde{G}} \longrightarrow \mathbb{Q} / \mathbb{Z}
$$

that come from an even $W$-invariant symmetric bilinear form

$$
\Lambda_{\text {coroots }} \times \Lambda_{\text {coroots }} \longrightarrow \mathbb{Z} \text {. }
$$

Let

$$
\Psi(G) \subset \Psi
$$

be the subgroup consisting of all elements $b$ such that $b\left(\pi_{1}(G) \times \pi_{1}(G)\right)=0$.

Given an element $\delta \in \pi_{1}(G)$, let

$$
\operatorname{ev}_{G}^{\delta}: \Psi(G) \longrightarrow \operatorname{Hom}\left(Z_{\widetilde{G}} / \pi_{1}(G), \mathbb{Q} / \mathbb{Z}\right)
$$

be the evaluation map that sends any $b$ to $b(\delta,-): Z_{G} \longrightarrow \mathbb{Q} / \mathbb{Z}$.

The following proposition (Proposition 7.4 in [BHf1]) computes the weight map.

Proposition 6.4. The kernel of the homomorphism

$$
p^{*}: \operatorname{Br}\left(M_{C}(G)^{\delta, \mathrm{rs}}\right) \longrightarrow \operatorname{Br}\left(\mathcal{M}_{C}(G)^{\delta}\right)
$$

is given by the $\operatorname{Coker}\left(\mathrm{ev}_{C}^{\delta}\right)$.

This proposition and Theorem 4.5 together imply the following:

Corollary 6.5. If $G$ is almost simple and simply connected, then $\operatorname{Br}\left(M_{C}(G)^{\mathrm{rs}}\right)=Z_{G}^{\vee}$. 


\section{A DIRECT APPROACH}

In this section we pursue the earlier spectral sequence argument.

Let $\widetilde{G} \longrightarrow G$ be the universal cover, where $G$ is semisimple. The kernel of this homomorphism is identified with $\pi_{1}(G)$. Define the finite group

$$
\Gamma:=H^{1}\left(C, \pi_{1}(G)\right) .
$$

Lemma 7.1. There is a short exact sequence

$$
0 \longrightarrow L_{C}(\widetilde{G}) / \pi_{1}(G) \longrightarrow L_{C}(G) \longrightarrow \Gamma \longrightarrow 0
$$

The quotient space $L_{C}(\widetilde{G}) / \pi_{1}(G)$ has the homotopy type of $G \times \Omega(\widetilde{G})^{2 g-1}$

Proof. Consider the short exact sequence of groups

$$
e \longrightarrow \pi_{1}(G) \longrightarrow \widetilde{G} \longrightarrow G \longrightarrow e .
$$

We have $H^{1}\left(C-p_{0}, G\right)=0\left[\mathrm{Ha}\right.$, also $H^{1}\left(C-p_{0}, \pi_{1}(G)\right)=H^{1}\left(C, \pi_{1}(G)\right)$. Therefore, from the long exact sequence of cohomologies associated to it, the exact sequence in the lemma is obtained.

The statement on homotopy type of $L_{C}(\widetilde{G}) / \pi_{1}(G)$ is a consequence of the fact that $L_{C}(\widetilde{G})$ itself has the homotopy type of $\widetilde{G} \times \Omega(\widetilde{G})^{2 g-1}$ (see Theorem 3.3).

Lemma 7.2. We have

(1) $H^{1}\left(B\left(L_{C}(\widetilde{G}) / \pi_{1}(G)\right), \mathbb{C}^{*}\right)=0$, and

(2) $H^{2}\left(B\left(L_{C}(\widetilde{G}) / \pi_{1}(G)\right), \mathbb{C}^{*}\right)=\pi_{1}(G)^{\vee}$.

Proof. Since $L_{C}(\widetilde{G})$ is connected (see Lemma 3.2),

$$
\pi_{1}\left(B\left(L_{C}(\widetilde{G}) / \pi_{1}(G)\right)\right)=\pi_{0}\left(L_{C}(\widetilde{G}) / \pi_{1}(G)\right)=0 .
$$

Hence the first statement follows.

To prove the second statement, consider the product decomposition

$$
B\left(L_{C}(\widetilde{G}) / \pi_{1}(G)\right)=B G \times B(\Omega \widetilde{G})^{2 g-1},
$$

and apply the Künneth decomposition to it. The individual cohomology computations are done as follows. We have $H^{1}\left(B G, \mathbb{C}^{*}\right)=0$ and $H^{1}\left(B(\Omega \widetilde{G}), \mathbb{C}^{*}\right)=0$ because $\pi_{1}(B G)=$ $\pi_{0}(G)=0 ;$ also,

$$
\pi_{1}(B(\Omega \widetilde{G}))=\pi_{0}(\Omega \widetilde{G})=\pi_{1}(\widetilde{G})=0 .
$$

These and Hurewicz isomorphism together imply that

$$
H^{2}\left(B(\Omega \widetilde{G}), \mathbb{C}^{*}\right) \cong \operatorname{Hom}\left(\pi_{2}(B(\Omega \widetilde{G})), \mathbb{C}^{*}\right) \text { and } H^{2}\left(B G, \mathbb{C}^{*}\right) \cong \operatorname{Hom}\left(\pi_{2}(B G), \mathbb{C}^{*}\right) .
$$

Since $\pi_{2}(B(\Omega \widetilde{G}))=\pi_{1}(\Omega \widetilde{G})=\pi_{2}(\widetilde{G})=0$, and $\pi_{2}(B G)=\pi_{1}(G)$, the second statement in the proposition follows.

The following is a generalization of Proposition 3.4.

Proposition 7.3. With the above notation and $\Gamma:=H^{1}\left(C, \pi_{1}(G)\right)$, 
(1) $H^{1}\left(B L_{C}(G), \mathbb{Z}\right)=0$, and

(2) there is a short exact sequence

$$
\left.0 \longrightarrow H^{2}\left(\Gamma, \mathbb{C}^{*}\right) \longrightarrow H^{2}\left(B L_{C}(G), \mathbb{C}^{*}\right)\right) \longrightarrow \pi_{1}(G)^{\vee} \longrightarrow 0
$$

Proof. The first part is a consequence of the fact that

$$
\pi_{1}\left(B L_{C}(G)\right)=\pi_{0}\left(L_{C}(G)\right)=\Gamma
$$

is a finite group.

For the second part, we use Lemma 7.1 to realize the space $B\left(L_{C}(\widetilde{G}) / \pi_{1}(G)\right)$ as a principal $\Gamma$-bundle over $B L_{C}(G)$. More precisely,

$$
B\left(L_{C}(\widetilde{G}) / \pi_{1}(G)\right)=\left(E L_{C}(G)\right) /\left(L_{C}(\widetilde{G}) / \pi_{1}(G)\right) \longrightarrow E L_{C}(G) / L_{C}(G)=B L_{C}(G) .
$$

The Serre spectral sequence gives the following exact sequence

$$
\begin{gathered}
H^{0}\left(\Gamma, H^{1}\left(B\left(L_{C}(\widetilde{G}) / \pi_{1}(G)\right), \mathbb{C}^{*}\right)\right) \longrightarrow H^{2}\left(\Gamma, \mathbb{C}^{*}\right) \longrightarrow \operatorname{kernel}\left[H^{2}\left(B L_{C}(G), \mathbb{C}^{*}\right) \rightarrow\right. \\
\left.H^{0}\left(\Gamma, H^{2}\left(B\left(L_{C}(\widetilde{G}) / \pi_{1}(G)\right), \mathbb{C}^{*}\right)\right)\right] \longrightarrow H^{1}\left(\Gamma, H^{1}\left(B\left(L_{C}(\widetilde{G}) / \pi_{1}(G)\right), \mathbb{C}^{*}\right)\right) .
\end{gathered}
$$

As $H^{1}\left(B\left(L_{C}(\widetilde{G}) / \pi_{1}(G)\right), \mathbb{C}^{*}\right)=0$ (see Lemma $7.2(1)$ ), this exact sequence reduces to an isomorphism

$$
H^{2}\left(\Gamma, \mathbb{C}^{*}\right) \stackrel{\sim}{\longrightarrow} \operatorname{kernel}\left[H^{2}\left(B L_{C}(G), \mathbb{C}^{*}\right) \rightarrow H^{0}\left(\Gamma, H^{2}\left(B\left(L_{C}(\widetilde{G}) / \pi_{1}(G)\right), \mathbb{C}^{*}\right)\right)\right] .
$$

We have $H^{0}\left(\Gamma, H^{2}\left(B\left(L_{C}(\widetilde{G}) / \pi_{1}(G)\right), \mathbb{C}^{*}\right)\right)=H^{2}\left(B\left(L_{C}(\widetilde{G}) / \pi_{1}(G)\right), \mathbb{C}^{*}\right)=\pi_{1}(G)^{\vee}$ by Lemma $\mathbf{7 . 2}(2)$. Therefore, to complete the proof it suffices to show that the homomorphism

$$
H^{2}\left(B L_{C}(G), \mathbb{C}^{*}\right) \longrightarrow H^{0}\left(\Gamma, H^{2}\left(B\left(L_{C}(\widetilde{G}) / \pi_{1}(G)\right), \mathbb{C}^{*}\right)\right)=\pi_{1}(G)^{\vee}
$$

in (7.2) is surjective.

Fix a point $x_{0} \in C \backslash\left\{p_{0}\right\}$, where $p_{0}$ is the base point in (3.1). Construct the group scheme

$$
\mathcal{H}:=L_{C}(G) \times{ }_{G} \widetilde{G}
$$

using the evaluation $L_{C}(G) \longrightarrow G$ at $x_{0}$. Both the natural morphisms

$$
B\left(L_{C}(\widetilde{G})\right) \stackrel{a}{\longrightarrow} B\left(L_{C}(\widetilde{G}) / \pi_{1}(G)\right) \text { and } B(\mathcal{H}) \stackrel{b}{\longrightarrow} B\left(L_{C}(G)\right)
$$

are gerbes banded by $\pi_{1}(G)$, while the natural morphism $B\left(L_{C}(\widetilde{G}) / \pi_{1}(G)\right) \stackrel{c}{\longrightarrow} B\left(L_{C}(G)\right)$ is an étale Galois covering with Galois group $\Gamma$. Note that we have a homomorphism

$$
L_{C}(\widetilde{G}) \longrightarrow \mathcal{H}=L_{C}(G) \times_{G} \widetilde{G}
$$

constructed using the evaluation $L_{C}(\widetilde{G}) \longrightarrow \widetilde{G}$ at $x_{0}$. This homomorphism produces a morphism

$$
d: B\left(L_{C}(\widetilde{G})\right) \longrightarrow B(\mathcal{H}),
$$

and the diagram

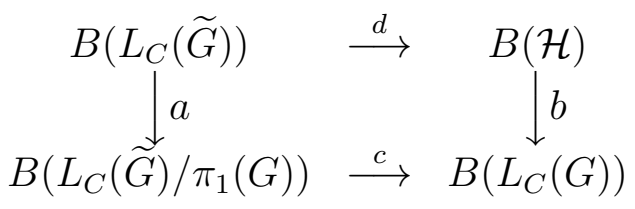


is clearly commutative. It is straight-forward to check that $d$ produces an isomorphism of the gerbe $B\left(L_{C}(\widetilde{G})\right)$ with the pulled back gerbe $c^{*} B(\mathcal{H})$.

The gerbe $B\left(L_{C}(\widetilde{G})\right) \stackrel{a}{\longrightarrow} B\left(L_{C}(\widetilde{G}) / \pi_{1}(G)\right)$ and a character of $\pi_{1}(G)$ together produce a gerbe on $B\left(L_{C}(\widetilde{G}) / \pi_{1}(G)\right)$ banded by $\mathbb{C}^{*}$. In view of Lemma $7.2(2)$, we know that all elements of $\left.H^{2}\left(B\left(L_{C}(\widetilde{G}) / \pi_{1}(G)\right), \mathbb{C}^{*}\right)\right)$ arise this way. Since the gerbe $B\left(L_{C}(\widetilde{G})\right) \stackrel{a}{\longrightarrow}$ $B\left(L_{C}(\widetilde{G}) / \pi_{1}(G)\right)$ is the pullback of a gerbe on $B\left(L_{C}(G)\right)$, it follows that the homomorphism in (7.3) is surjective.

The analogue of Theorem 4.1 for a general semisimple group is the following.

Theorem 7.4. For any $\delta \in \pi_{1}(G)$, the following exact sequence computes the Brauer group of the moduli stack of principal $G$-bundles over $C$ :

$$
\operatorname{Pic}\left(\mathcal{M}_{C}(G)^{\delta}\right) \longrightarrow \operatorname{Pic}\left(\mathcal{Q}_{\widetilde{G}}\right) \longrightarrow H^{2}\left(B L_{C}(G), \mathbb{C}^{*}\right) \longrightarrow \operatorname{Br}\left(\mathcal{M}_{C}(G)^{\delta}\right) \longrightarrow 0 ;
$$

the above group $H^{2}\left(B L_{C}(G), \mathbb{C}^{*}\right)$ is computed by the exact sequence in (7.1).

Proof. The proof of the theorem follows the same steps as in the proof of Theorem 4.1 , namely the descent spectral sequence and the cohomology computations - which are now provided by Proposition 7.3 .

As the last step in the computation we prove the following.

Proposition 7.5. The homomorphism

$$
p^{*}: \operatorname{Br}\left(M_{C}(G)^{\delta, \text { rs }}\right) \longrightarrow \operatorname{Br}\left(\mathcal{M}_{C}(G)^{\delta, \text { rs }}\right)=\operatorname{Br}\left(\mathcal{M}_{C}(G)^{\delta}\right)
$$

in (6.8) is surjective.

Proof. The above equality $\operatorname{Br}\left(\mathcal{M}_{C}(G)^{\delta \text {,rs }}\right)=\operatorname{Br}\left(\mathcal{M}_{C}(G)^{\delta}\right)$ follows immediately from Proposition 4.3 .

Let $\widetilde{G} \longrightarrow G$ be the universal covering homomorphism; its kernel is $\pi_{1}(G)$. We get an inclusion $\pi_{1}(G) \subset Z_{\widetilde{G}}$, and the quotient is identified with $Z_{G}$. There is a canonical inclusion of $Z_{G}$

(see Lemma 7.1).

$$
Z_{G} \hookrightarrow L_{C}(\widetilde{G}) / \pi_{1}(G) \hookrightarrow L_{C}(G)
$$

Let

$$
\mathcal{Q}_{1} \subset \mathcal{Q}_{\widetilde{G}}
$$

be the open sub-stack defined by the inverse image of $\mathcal{M}_{C}(G)^{\delta \text {,rs }}$ under the morphism $q_{G}^{\delta}$ in (6.1). Choosing an element $\zeta \in(L G)^{\delta}(\mathbb{C})$ as in Proposition 6.1, we get an action of the group $L_{C}(G) / Z_{G}$ on $\mathcal{Q}_{1}$ such that the corresponding quotient is $M_{C}(G)^{\delta \text {,rs }}$.

Consider the morphism $p$ in (6.7). Since the fiber product $\mathcal{Q}_{1} \times_{M_{C}(G)^{\delta, \text { rs }}} \mathcal{M}_{C}(G)^{\delta \text {,rs }}$ is identified with $\mathcal{Q}_{1} \times Z(G)$, we conclude, by taking cohomologies, that the diagram

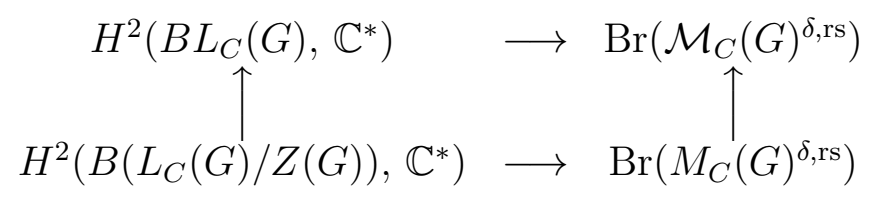


is commutative. The upper horizontal arrow is surjective by Theorem 7.4 . In view of the above diagram, to complete the proof of the proposition it suffices to show that the homomorphism

$$
H^{2}\left(B\left(L_{C}(G) / Z(G)\right), \mathbb{C}^{*}\right) \longrightarrow H^{2}\left(B L_{C}(G), \mathbb{C}^{*}\right)
$$

is surjective. But this surjectivity follows by applying Proposition $7.3(2)$ to $B L_{C}(G)$ and $B\left(L_{C}(G) / Z(G)\right)$ and observing that the terms in the resulting two exact sequences match. This completes the proof of the proposition.

Combining the above proposition with Proposition 6.4 and Lemma 4.4 we get the following.

Corollary 7.6. For any semisimple $G$, there is a short exact sequence

$$
0 \longrightarrow \operatorname{Coker}\left(\mathrm{ev}_{G}^{\delta}\right) \longrightarrow \operatorname{Br}\left(M_{C}(G)^{\delta, \mathrm{rs}}\right) \longrightarrow \operatorname{Br}\left(\mathcal{M}_{C}(G)^{\delta}\right) \longrightarrow 0 \text {. }
$$

\section{Computations For Classical groups}

8.1. The cases of $\mathrm{SL}_{n}$ and $\mathrm{PGL}_{n}$. Take a positive integer $n$, and take any $d \in[0, n-1]$. Fix a line bundle $L_{d}$ on $C$ of degree $d$. The twisted moduli stack $\mathcal{M}_{C}^{d}\left(\mathrm{SL}_{n}\right)$ is the moduli stack of vector bundles on $C$ of rank $n$ and determinant isomorphic to $L_{d}$. Theorem 4.1 and Theorem 5.2 imply that

$$
\operatorname{Br}\left(\mathcal{M}_{C}^{d}\left(\mathrm{SL}_{n}\right)\right)=0
$$

for any $d$.

By Proposition 6.4, the Brauer group of $M_{C}^{d}\left(\mathrm{SL}_{n}\right)^{\mathrm{rs}}$ coincides with the cokernel of the homomorphism $\operatorname{ev}_{G}^{\delta}$, and this can be computed to be $\mathbb{Z} /$ g.c.d. $(n, d) \mathbb{Z}$ by Table 1 of [BHf1]. This recovers the results of [BBGN].

For the case of $\mathrm{PGL}_{n}$, the exact sequence given in Theorem 6.3 or Theorem 7.4 reproduces Theorem 1 of $[\mathrm{BHg}$.

The above approach also works for quotients of $\mathrm{SL}_{n}$ by finite subgroups of the center $Z_{\mathrm{SL}_{n}}$.

8.2. The cases $\operatorname{Sp}_{2 n}$ and $\operatorname{PSp}_{2 n}$. We have $\operatorname{Br}\left(\mathcal{M}_{C}^{d}\left(\operatorname{Sp}_{2 n}\right)\right)=0$ for $d=0,1$ by Theorem 4.1 and Theorem 5.2 .

From Corollary 6.5 we have $\operatorname{Br}\left(M_{C}^{0}\left(\mathrm{Sp}_{2 n}\right)^{\mathrm{rs}}\right)=\mathbb{Z} / 2 \mathbb{Z}$.

Proposition 8.1. Let $d=1$.

(1) Assume that $n \geq 3$ is odd. Then

$$
\operatorname{Br}\left(M_{C}^{1}\left(\mathrm{Sp}_{2 n}\right)^{\mathrm{rs}}\right)=0 .
$$

The square of the generating line bundle on $\mathcal{M}_{C}^{1}\left(\mathrm{Sp}_{2 n}\right)$ descends to $M_{C}^{1}\left(\mathrm{Sp}_{2 n}\right)^{\mathrm{rs}}$ and generates $\operatorname{Pic}\left(M_{C}^{1}\left(\operatorname{Sp}_{2 n}\right)\right)^{\mathrm{rs}}$.

(2) Assume that $n \geq 3$ is even. Then $\operatorname{Br}\left(M_{C}^{1}\left(\operatorname{Sp}_{2 n}\right)^{\mathrm{rs}}\right)=\mathbb{Z} / 2 \mathbb{Z}$. The generating line bundle on $\mathcal{M}_{C}^{1}\left(\operatorname{Sp}_{2 n}\right)$ descends to $M_{C}^{1}\left(\operatorname{Sp}_{2 n}\right)^{\mathrm{rs}}$ and generates $\operatorname{Pic}\left(M_{C}^{1}\left(\operatorname{Sp}_{2 n}\right)^{\mathrm{rs}}\right)$. 
Proof. This follows by the combination of exact sequences in Theorem 5.4 and Proposition 6.4. The cokernel of the homomorphism $\mathrm{ev}_{G}^{\delta}$ is 0 when $n$ is odd, and it is $\mathbb{Z} / 2 \mathbb{Z}$ when $n$ is even by Table 1 of [BHf1].

Corollary 8.2. Let $M_{C}^{1}\left(\mathrm{Sp}_{2 n}\right)^{\mathrm{ss}}$ be the twisted moduli space of semistable $\mathrm{Sp}_{2 n}$-bundles. The variety $M_{C}^{1}\left(\mathrm{Sp}_{2 n}\right)^{\mathrm{ss}}$ is locally factorial for odd $n$, and it is not locally factorial for even $n$.

Proof. The Picard group of $M_{C}^{1}\left(\mathrm{Sp}_{2 n}\right)^{\mathrm{ss}}$ is always generated by the descent of the square of the generating line bundle on $\mathcal{M}_{C}^{1}\left(\mathrm{Sp}_{2 n}\right)$ (see [BLS, p. 209, Proposition 11.2(a)]). Therefore, the corollary follows from Proposition 8.1 .

Note that $\pi_{1}\left(\mathrm{PSp}_{2 n}\right)=Z_{S p_{2 n}}=\mathbb{Z} / 2 \mathbb{Z}$. For $d \in \pi_{1}\left(\mathrm{PSp}_{2 n}\right)$, let

$$
\mathcal{M}_{C}\left(\mathrm{PSp}_{2 n}\right)^{d} \subset \mathcal{M}_{C}\left(\mathrm{PSp}_{2 n}\right)
$$

be the connected component of the moduli stack of principal $\mathrm{PSp}_{2 n}$-bundles corresponding to $d$. Similarly,

$$
M_{C}\left(\mathrm{PSp}_{2 n}\right)^{d, \mathrm{rs}} \subset M_{C}\left(\mathrm{PSp}_{2 n}\right)^{\mathrm{rs}}
$$

is the connected component of the moduli space corresponding to the element $d$. The locus $\mathcal{M}_{C}\left(\mathrm{PSp}_{2 n}\right)^{d, \mathrm{rs}}$ in $\mathcal{M}_{C}\left(\mathrm{PSp}_{2 n}\right)^{d}$ of regularly stable bundles is an open sub-stack such that the complement is of codimension at least two. Hence the Brauer groups of $\mathcal{M}_{C}\left(\mathrm{PSp}_{2 n}\right)^{d}$ and $\mathcal{M}_{C}\left(\mathrm{PSp}_{2 n}\right)^{d, \mathrm{rs}}$ coincide.

Define

$$
\Gamma:=H^{2}\left(C, \mu_{2}\right)
$$

(the 2-torsion points of the Jacobian of $C$ ).

Proposition 8.3. Let $d=0$ or 1 . If $n \geq 3$ is odd, then $\operatorname{Br}\left(\mathcal{M}_{C}\left(\mathrm{PSp}_{2 n}\right)^{d}\right) \cong H^{2}\left(\Gamma, \mathbb{C}^{*}\right)$. If $n \geq 3$ is even, then $\operatorname{Br}\left(\mathcal{M}_{C}\left(\operatorname{PSp}_{2 n}\right)^{d}\right) \cong H^{2}\left(\Gamma, \mathbb{C}^{*}\right) \oplus(\mathbb{Z} / 2 \mathbb{Z})$.

Proof. Let $U^{d, \mathrm{rs}} \subset M_{C}^{d}\left(\mathrm{Sp}_{2 n}\right)^{\mathrm{rs}}$ be the Zariski open subset defined by the inverse image of $M_{C}\left(\mathrm{PSp}_{2 n}\right)^{d, \mathrm{rs}}$. The codimension of the complement of $U^{d, \mathrm{rs}}$ is at least two. To prove the proposition one can use both the approaches used here. For example, Theorem 6.3 gives the following exact sequence

$$
0 \longrightarrow \mathbb{Z} / m \mathbb{Z} \longrightarrow H^{2}\left(\Gamma, \mathbb{C}^{*}\right) \longrightarrow \operatorname{Br}\left(M_{C}\left(\mathrm{PSp}_{2 n}\right)^{d, \mathrm{rs}}\right) \longrightarrow \operatorname{Br}\left(U^{d, \mathrm{rs}}\right) \longrightarrow 0,
$$

where $m$ is the smallest power of the generating line bundle on $U^{d, \mathrm{rs}}$ which descends to the moduli space $M_{C}\left(\mathrm{PSp}_{2 n}\right)^{d, \mathrm{rs}}$.

Since $M_{C}^{d}\left(\mathrm{Sp}_{2 n}\right)^{\mathrm{rs}}$ is smooth, and the codimension of the complement of $U^{d, \mathrm{rs}}$ is at least two, the homomorphisms

$$
\operatorname{Pic}\left(M_{C}^{d}\left(\mathrm{Sp}_{2 n}\right)^{\mathrm{rs}}\right) \longrightarrow \operatorname{Pic}\left(U^{d, \mathrm{rs}}\right) \text { and } \operatorname{Br}\left(M_{C}^{d}\left(\mathrm{Sp}_{2 n}\right)^{\mathrm{rs}}\right) \longrightarrow \operatorname{Br}\left(U^{d, \mathrm{rs}}\right)
$$

induced by the inclusion $U^{d, \mathrm{rs}} \hookrightarrow M_{C}^{d}\left(\mathrm{Sp}_{2 n}\right)^{\mathrm{rs}}$ are isomorphisms. 
When $n$ is even, by [BLS, p. 191, Proposition 4.2], the generating line bundle on the affine Grassmannian $\mathcal{Q}_{\mathrm{Sp}_{2 n}}$ descends all the way to $M_{C}\left(\mathrm{PSp}_{2 n}\right)^{d, \mathrm{rs}}$. Hence in this case we have $m=1$ in (8.1).

When $d=0$ with $n$ odd, the generating line bundle on the affine Grassmannian descends to $M_{C}^{0}\left(\mathrm{Sp}_{2 n}\right)^{\mathrm{rs}}$ (see Proposition [8.1), but it does not descend to $M_{C}\left(\mathrm{PSp}_{2 n}\right)^{0, \text { rs }}$ by [BLS, Proposition 4.2]. Hence in this case we have $m=2$ in (8.1).

If $d=1$ with $n$ odd, then the smallest power of the generating line bundle on the affine Grassmannian which descends to $M_{C}^{1}\left(\mathrm{Sp}_{2 n}\right)^{\text {rs }}$ is 2 , and also 2 is the smallest power of the generating line bundle on the affine Grassmannian that descends to $M_{C}\left(\mathrm{PSp}_{2 n}\right)^{0, \text { rs }}$ (again by Proposition 8.1 and [BLS, Proposition 4.2]). Hence $m=1$ in this case.

If $d=1$ with $n$ odd, then the above observation and Proposition 8.1 together imply the proposition.

In the remaining cases we get a short exact sequence of the form

$$
0 \longrightarrow(\mathbb{Z} / 2 \mathbb{Z})^{b} \longrightarrow \operatorname{Br}\left(M_{C}\left(\mathrm{PSp}_{2 n}\right)^{d, \mathrm{rs}}\right) \longrightarrow \mathbb{Z} / 2 \mathbb{Z} \longrightarrow 0
$$

To complete the proof of the proposition it suffices to show that the sequence in (8.2) splits. The exact sequence splits if the generating Brauer class of $M_{C}^{d}\left(\mathrm{Sp}_{2 n}\right)^{\mathrm{rs}}$ (when it is non-trivial) is the pull back of a two-torsion class in $\operatorname{Br}\left(M_{C}\left(\mathrm{PSp}_{2 n}\right)^{d, \mathrm{rs}}\right)$. But this was proved in Section 66 (see the proof that the homomorphism in (6.6) is surjective).

8.3. The cases of $\operatorname{Spin}_{n}, \mathrm{SO}_{n}$ and $\mathrm{PSO}_{n}$. The center $Z_{\operatorname{Spin}_{n}}$ of $\operatorname{Spin}_{n}$ is $\mathbb{Z} / 2 \mathbb{Z}$ when $n$ odd, and it is $\mathbb{Z} / 4 \mathbb{Z}$ or $\mathbb{Z} / 2 \mathbb{Z} \times \mathbb{Z} / 2 \mathbb{Z}$ depending on whether $n$ is of the form $4 l+2$ or $4 l$. Take any $\delta \in Z_{\operatorname{Spin}_{n}}$. Since the Dynkin index of the standard representation of $\operatorname{Spin}_{n}$ in $\mathrm{SL}_{n}$ is 2 , the Picard group of the twisted moduli stack $\mathcal{M}_{C}^{\delta}\left(\operatorname{Spin}_{n}\right)$ is generated by the Pffafian line bundle $P$ whose square is the determinant bundle of cohomology.

By Theorem 4.1 and Theorem 5.2 ,

$$
\operatorname{Br}\left(\mathcal{M}_{C}^{\delta}\left(\operatorname{Spin}_{n}\right)\right)=0 .
$$

Proposition 8.4. (1) If $\delta \in Z_{\operatorname{Spin}_{\mathrm{n}}}$ is zero, then $\operatorname{Br}\left(M_{C}^{0}\left(\operatorname{Spin}_{n}\right)^{\mathrm{rs}}\right)=Z_{\operatorname{Spin}_{n}}^{\mathrm{V}}$.

(2) Assume that $n \geq 4$ is odd. Then $\operatorname{Br}\left(M_{C}^{1}\left(\operatorname{Spin}_{n}\right)^{\mathrm{rs}}\right)=\mathbb{Z} / 2 \mathbb{Z}$. The Pffafian bundle descends to $M_{C}^{1}\left(\operatorname{Spin}_{n}\right)^{\mathrm{rs}}$ and generates the Picard group $\operatorname{Pic}\left(M_{C}^{1}\left(\operatorname{Spin}_{n}\right)^{\mathrm{rs}}\right)$.

(3) Assume that $n=4 l+2 \geq 8$ and $\delta=1$ or $3 \bmod (4)$. Then $\operatorname{Br}\left(M_{C}^{\delta}\left(\operatorname{Spin}_{n}\right)^{\mathrm{rs}}\right)=$ 0 . The fourth power of the Pffafian bundle descends to $M_{C}^{\delta}\left(\operatorname{Spin}_{n}\right)^{\mathrm{rs}}$ and it generates the Picard group $\operatorname{Pic}\left(M_{C}^{\delta}\left(\operatorname{Spin}_{n}\right)^{\mathrm{rs}}\right)$.

(4) Assume that $n=4 l+2 \geq 8$ and $\delta=2 \bmod (4)$. Then $\operatorname{Br}\left(M_{C}^{\delta}\left(\operatorname{Spin}_{n}\right)^{\mathrm{rs}}\right)=$ $\mathbb{Z} / 2 \mathbb{Z}$. The square of the Pffafian bundle descends to $M_{C}^{\delta}\left(\operatorname{Spin}_{n}\right)^{\mathrm{rs}}$ generating $\operatorname{Pic}\left(M_{C}^{\delta}\left(\operatorname{Spin}_{n}\right)^{\mathrm{rs}}\right)$.

(5) Assume that $n=4 l \geq 8$ and $\delta \neq 0$. Then $\operatorname{Br}\left(M_{C}^{\delta}\left(\operatorname{Spin}_{n}\right)^{\mathrm{rs}}\right)=\mathbb{Z} / 2 \mathbb{Z}$. The square of the Pffafian bundle descends to $M_{C}^{\delta}\left(\operatorname{Spin}_{n}\right)^{\mathrm{rs}}$ generating the Picard group.

Proof. In view of Proposition 6.4, this is a straight-forward calculation using Table 1 in BHf1. 
For the case of $\mathrm{SO}_{n}$, note that $\pi_{1}\left(\mathrm{SO}_{n}\right) \subset Z_{\mathrm{Spin}_{n}}$. For any $\delta \in \pi_{1}\left(\mathrm{SO}_{n}\right)$, let

$$
\mathcal{M}_{C}\left(\mathrm{SO}_{n}\right)^{\delta} \subset \mathcal{M}_{C}\left(\mathrm{SO}_{n}\right)
$$

be the connected component corresponding to $\delta$. Similarly, let

$$
M_{C}\left(\mathrm{SO}_{n}\right)^{\delta, \mathrm{rs}} \subset M_{C}\left(\mathrm{SO}_{n}\right)^{\mathrm{rs}}
$$

be the connected component corresponding to $\delta$.

Define

$$
\Gamma^{\prime}:=H^{1}\left(C, \pi_{1}\left(\mathrm{SO}_{n}\right)^{\vee}\right) .
$$

Proposition 8.5. Let $n \geq 8$. Take any $\delta \in \pi_{1}\left(\mathrm{SO}_{n}\right)$.

(1) If $\delta=0$, then

$$
\operatorname{Br}\left(M_{C}\left(\mathrm{SO}_{n}\right)^{0, \mathrm{rs}}\right)=H^{2}\left(\Gamma^{\prime}, \mathbb{C}^{*}\right) \oplus Z_{\mathrm{Spin}_{n}}^{\vee}
$$

(2) If $\delta \neq 0$, then $\operatorname{Br}\left(M_{C}\left(\mathrm{SO}_{n}\right)^{\delta, \mathrm{rs}}\right) \cong H^{2}\left(\Gamma^{\prime}, \mathbb{C}^{*}\right) \oplus \mathbb{Z} / 2 \mathbb{Z}$.

(3) We have $\operatorname{Br}\left(\mathcal{M}_{C}\left(\mathrm{SO}_{n}\right)^{\delta}\right) \cong H^{2}\left(\Gamma^{\prime}, \mathbb{C}^{*}\right) \oplus \mathbb{Z} / 2 \mathbb{Z}$.

Proof. The proof is very similar to that for $\mathrm{Sp}_{n}$ as done in Proposition 8.1 .

For any $\delta \in \pi_{1}\left(\mathrm{SO}_{n}\right)$, let

$$
U^{\delta, \mathrm{rs}} \subset M_{C}^{\delta}\left(\operatorname{Spin}_{n}\right)^{\mathrm{rs}}
$$

be the Zariski open subset given by the inverse image of $M_{C}\left(\mathrm{SO}_{n}\right)^{\delta, \mathrm{rs}}$. The codimension of the complement of $U^{\delta, \mathrm{rs}}$ in $M_{C}^{\delta}\left(\operatorname{Spin}_{n}\right)$ is at least two. Now Theorem 6.3 gives the exact sequence

$$
0 \longrightarrow \mathbb{Z} / m \mathbb{Z} \longrightarrow H^{2}\left(\Gamma^{\prime}, \mathbb{C}^{*}\right) \longrightarrow \operatorname{Br}\left(M_{C}\left(\mathrm{SO}_{n}\right)^{\delta, \mathrm{rs}}\right) \longrightarrow \operatorname{Br}\left(U^{\delta, \mathrm{rs}}\right) \longrightarrow 0,
$$

where $m$ is the smallest power of the generating line bundle on $U^{\delta, \mathrm{rs}}$ which descends to $M_{C}\left(\mathrm{SO}_{2}\right)^{\delta, \mathrm{rs}}$.

We will show that the integer $m$ defined in (8.3) is 1 . This amounts to showing that the generating line bundle on $M_{C}^{\delta}\left(\operatorname{Spin}_{n}\right)^{\mathrm{rs}}$ descend to the quotient $M_{C}\left(\mathrm{SO}_{n}\right)^{\delta, \mathrm{rs}}=U^{\delta, \mathrm{rs}} / \Gamma^{\prime}$.

If $\delta=0$, this follows from the existence of the Pffafian bundle.

Assume that $\delta \neq 0$. We note that Proposition 8.4 gives the smallest power of the Pffafian bundle which descends to $M_{C}^{\delta}\left(\operatorname{Spin}_{n}\right)^{\text {rs }}$ from the moduli stack $\mathcal{M}_{C}^{\delta}\left(\operatorname{Spin}_{n}\right)$. Hence it is enough to compute the smallest power of the Pffafian bundle on the moduli stack $\mathcal{M}_{C}^{\delta}\left(\operatorname{Spin}_{n}\right)$ that descends to $M_{C}\left(\mathrm{SO}_{n}\right)^{\delta, \mathrm{rs}}$. To calculate this, we again use Proposition 6.4 and the Table 1 of [BHf1]; we explicitly check that the smallest power of the Pffafian bundle that descends to $M_{C}\left(\mathrm{SO}_{n}\right)^{\delta \text {,rs }}$ coincide with the power that descends to $M_{C}^{\delta}\left(\operatorname{Spin}_{n}\right)^{\mathrm{rs}}$.

Let

$$
0 \longrightarrow H^{2}\left(\Gamma^{\prime}, \mathbb{C}^{*}\right) \longrightarrow \operatorname{Br}\left(M_{C}\left(\mathrm{SO}_{n}\right)^{\delta, \mathrm{rs}}\right) \longrightarrow Z_{\operatorname{Spin}_{n}} \longrightarrow 0,
$$

be the exact sequence obtained from (8.3)). 
To conclude parts (1) and (2) of the proposition, we still need to show that the exact sequence in (8.4) splits. The proof of this splitting is identical to the case for $\mathrm{Sp}_{n}$; the details are omitted.

For the last part of the proposition, we use the existence of Pffafian bundle to conclude that the homomorphism

$$
H^{2}\left(B L_{C}\left(\mathrm{SO}_{n}\right), \mathbb{C}^{*}\right) \longrightarrow \operatorname{Br}\left(\mathcal{M}_{C}\left(\mathrm{SO}_{n}\right)^{\delta}\right)
$$

in Theorem 7.4 is an isomorphism. Now Proposition 7.3 gives an exact sequence

$$
0 \longrightarrow H^{2}\left(\Gamma^{\prime}, \mathbb{C}^{*}\right) \longrightarrow \operatorname{Br}\left(\mathcal{M}_{C}\left(\mathrm{SO}_{n}\right)^{\delta}\right) \longrightarrow \mathbb{Z} / 2 \mathbb{Z} \longrightarrow 0
$$

This sequence splits by using the argument for splitting of the exact sequence in (8.4) and Proposition 7.5 .

For the case of $\mathrm{PSO}_{2 n}$, the components of the moduli space are parametrized by the center $Z_{\mathrm{Spin}_{2 n}}$. Since $\mathrm{PSO}_{2 n}$ is of adjoint type, the Brauer groups of $M_{C}\left(\mathrm{PSO}_{2 n}\right)^{\delta \text {,rs }}$ and $\mathcal{M}_{C}\left(\mathrm{PSO}_{2 n}\right)^{\delta}$ can be identified as before. Define

$$
\Gamma_{1}:=H^{1}\left(C, Z_{\operatorname{Spin}_{2 n}}^{\vee}\right) \text { and } B=H^{2}\left(\Gamma_{1}, \mathbb{C}^{*}\right)
$$

Using the above methods and the description of the Picard and Brauer groups as done in Proposition 8.4 and [BLS, Proposition 5.5], we get the following proposition.

Proposition 8.6. Let $n \geq 4$. We have $\operatorname{Br}\left(M_{C}\left(\mathrm{PSO}_{2 n}\right)^{\delta, \mathrm{rs}}\right)=\left(B / A_{2 n, \delta}\right) \oplus Z^{\vee}$, where the groups $A_{2 n, \delta}$ is computed as follows: $A_{4 n, \delta}=\mathbb{Z} / 2 \mathbb{Z}$ (respectively, $A_{4 n, \delta}=0$ ) if $\delta=0$ (respectively, $\neq 0$ ), and $A_{4 n+2, \delta}=\mathbb{Z} / 4 \mathbb{Z}$ (respectively, $A_{4 n+2, \delta}=\mathbb{Z} / 2 \mathbb{Z}$ ) if $\delta$ is $0 \bmod (4)$ (respectively, $2 \bmod (4)$ ), and $A_{4 n+2, \delta}=0$ otherwise.

There is a remaining classical group $\Omega_{4 n}$ for $n \geq 3$ defined by taking quotient of $\operatorname{Spin}_{4 n}$ by a central subgroup of order 2 different from the one that defines $\mathrm{SO}_{4 n}$. There are two choices for such a sub-group and they define isomorphic groups.

We can use the methods described above to determine the Picard and Brauer groups of the moduli spaces of $\Omega_{4 n}$ bundles, and we get the following.

Proposition 8.7. (1) We have $\operatorname{Br}\left(M_{C}\left(\Omega_{4 n}\right)^{\delta, \text { rs }}\right) \cong H^{2}\left(\Gamma, \mathbb{C}^{*}\right) \oplus \mathbb{Z} / 2 \mathbb{Z}$. The square of the Pffafian bundle descends to $M_{C}\left(\Omega_{4 n}\right)^{\delta, \mathrm{rs}}$ generating the Picard group.

(2) We have $\operatorname{Br}\left(\mathcal{M}_{C}\left(\Omega_{4 n}\right)^{\delta}\right) \cong H^{2}\left(\Gamma, \mathbb{C}^{*}\right) \oplus A_{4 n, d}$, where $A_{4 n, d}=0$ if either $d=0$ or $n$ is odd, and $A_{4 n, d}=\mathbb{Z} / 2 \mathbb{Z}$ otherwise. The Picard group of $\mathcal{M}_{C}\left(\Omega_{4 n}^{\delta}\right)$ is generated by the descent of the Pffafian bundle if $n$ is even with $d \neq 0$, and in other cases $\operatorname{Pic}\left(\mathcal{M}_{C}\left(\Omega_{4 n}^{\delta}\right)\right)$ is generated by the descent of the square of the Pffafian bundle.

The two exceptional groups $E_{6}$ and $E_{7}$ have nontrivial center. Using Theorem 7.4 and Corollary 7.6 the Brauer group of the moduli space and the moduli stack for corresponding adjoint type groups can be computed as above. 


\section{REFERENCES}

[BBGN] V. Balaji, I. Biswas, O. Gabber and D. S. Nagaraj, Brauer obstruction for a universal vector bundle, Comp. Ren. l'Acad. Sci. (Paris) - Math. 345 (2007), 265-268.

[BLS] A. Beauville, Y. Laszlo and C. Sorger, The Picard group of the moduli of $G$-bundles on a curve, Compositio Math. 112 (1998), 183-216.

[BHg] I. Biswas and A. Hogadi, Brauer group of moduli spaces of $\operatorname{PGL}(r)$-bundles over a curve, Adv. Math. 225 (2010), 2317-2331.

[BHf1] I. Biswas and N. Hoffmann, Poincaré families of G-bundles on a curve, Math. Ann. (to appear), http://arxiv.org/abs/1001.2123.

[BHf2] I. Biswas and N. Hoffmann, A Torelli theorem for moduli spaces of principal bundles over a curve, Ann. Inst. Fourier (to appear), http://arxiv.org/abs/1003.4061.

[dJ] A. J. de Jong, A result of Gabber, http://www.math.columbia.edu/ dejong/papers/2-gabber.pdf.

[Fa] G. Faltings, Stable G-bundles and projective connections, Jour. Alg. Geom. 2 (1993), 507-568.

[Ha] G. Harder, Halbeinfache Gruppenschemata über Dedekindringen, Invent. Math. 4 (1967), 165191.

[KN] S. Kumar and M. S. Narasimhan, Picard group of the moduli spaces of G-bundles, Math. Ann. 308 (1997), 155-173.

[Me] A. S. Merkur'ev, A seven-term sequence in the Galois theory of schemes, Math. USSR Sb. 37 (1980), 367-380, http://iopscience.iop.org/0025-5734/37/3/A05/pdf/0025-5734_37_3_A05.pdf.

[Mi] J. S. Milne, Étale cohomology, Princeton Mathematical Series, 33. Princeton University Press, Princeton, N.J., 1980.

[Ne] P. E. Newstead, Comparison theorems for conic bundles, Math. Proc. Cambridge Philos. Soc. 90 (1981), 21-31.

[Ra] A. Ramanathan, Moduli for principal bundles over algebraic curves. I, Proc. Indian Acad. Sci. (Math. Sci.) 106 (1996), 301-328.

[Te] C. Teleman, Borel-Weil-Bott theory on the moduli stack of $G$-bundles over a curve, Invent. Math. 134 (1998), 1-57.

School of Mathematics, Tata Institute of Fundamental Research, Homi Bhabha Road, BOMBAY 400005, INDIA

E-mail address: indranil@math.tifr.res.in

School of Mathematics, Tata Institute of Fundamental Research, Homi Bhabha Road, BOMBAY 400005, INDIA

E-mail address: yogi@math.tifr.res.in 